\title{
Adaptive consensus of multi-agent in networks with jointly connected topologies *
}

\author{
Hui Yu ${ }^{a, b, \star \star}$, Xiaohua Xia ${ }^{b}$ \\ ${ }^{a}$ College of Science, China Three Gorges University, Yichang 443002, China \\ ${ }^{\mathrm{b}}$ Centre of New Energy Systems, Department of Electrical, Electronics and Computer Engineering, \\ University of Pretoria, Pretoria 0002, South Africa
}

\begin{abstract}
In this paper, the consensus problem of multi-agent following a leader is studied. An adaptive design method is presented for multi-agent systems with non-identical unknown nonlinear dynamics, and for a leader to be followed that is also nonlinear and unknown. By parameterizations of unknown nonlinear dynamics of all agents, a decentralized adaptive consensus algorithm is proposed in networks with jointly connected topologies by incorporating local consensus errors in addition to relative position feedback. Analysis of stability and parameter convergence of the proposed algorithm are conducted based on algebraic graph theory and Lyapunov theory. Finally, examples are provided to validate the theoretical results.
\end{abstract}

Key words: Consensus; Adaptive control; Parameter convergence; Jointly connected topology; Multi-agent systems.

\section{Introduction}

Distributed coordination of a group of dynamical agents is of interest in control and robotics. This is due to the broad applications of multi-agent systems in many areas, e.g., in multi-vehicle rendezvous, formation control of multi-robots, flocking, swarming, distributed sensor fusion, attitude alignment, and congestion control in communication networks. An important problem in distributed coordinated networks of dynamical agents is to find a distributed control law so that all agents can reach consensus on a common decision value. This problem is the so-called consensus problem.

Early well-known works on consensus coordination for networks of dynamical agents have been done in the context of control theory in Jadbabaie et al. (2003); Fax and Murray (2004); Lin et al. (2004); Olfati-Saber and

\footnotetext{
ऋ This work is supported by Nature Science Foundation of China (61074091, 61174216 and 61174177), Nature Science Foundation of Hubei Province (2011CDB187), Scientific Innovation Team Project of Hubei Provincial College (T200809 and T201103), Natural Science Research Project of the Education Department of Hubei Province (D20101201). This paper was not presented at any conference.

${ }^{\star \star}$ Corresponding author. Tel.: +86 7176392618.

Email addresses: yuhui@ctgu.edu.cn (Hui Yu), xxia@postino.up.ac.za (Xiaohua Xia).
}

Murray (2004); Savkin (2004); Ren and Beard (2005); Moreau (2005); Hatano and Mesbahi (2005), to name just a few. In recent years, relevant topics on consensus problem have been extensively further investigated in different situations, for example, consensus in networks with time-delays (Sun and Wang, 2009; Zhu and Cheng, 2010), finite-time consensus (Khoo et al., 2009), consensus in stochastic networks (Tahbaz-Salehi and Jadbabaie, 2008), quantized consensus (Kashyap et al., 2007), etc.

Recently, an interesting topic is the consensus problem of a group of agents with unknown information. In Hong et al. (2006), the authors proposed a consensus algorithm of agents with an active leader with unmeasurable state and variable interactive topology. The algorithm is also extended to the case that the interconnected graphs of agents are not always connected in intervals with identical length. In Bai et al. (2008, 2009), the authors studied a coordination problem steering a group of agents to a formation that translates with a prescribed reference velocity. Decentralized adaptive designs are proposed for reference velocity recovery using relative position feedback in Bai et al. (2008) and tracking of the reference velocity by incorporating relative velocity feedback in addition to relative position feedback in Bai et al. (2009). In Hou et al. (2009), the authors proposed a robust decentralized adaptive control approach using neural net- 
work to solve consensus problem of multi-agents with uncertainties and external disturbances in undirected networks. In Das and Lewis (2010), the authors presented a design method for adaptive synchronization controller$\mathrm{s}$ for distributed systems having non-identical unknown nonlinear dynamics, and for a target dynamics to be tracked that is also nonlinear and unknown. Under some assumptions, the authors proved that the overall local cooperative error vector and the neural network weight estimation errors are both uniformly ultimately bounded. In Yu et al. (2009b), for an unknown regulatory network with time delay and uncertain noise disturbance, an adaptive filtering approach is proposed to ensure the stochastic stability of the error states between the unknown network and the estimated model. Other kinds of adaptive synchronization design of complex dynamical networks are by using adaptive tuning of the coupling strength (Yu et al., 2009a), network weights, etc.

In this paper, we consider the adaptive consensus coordination problem of a group of agents with non-identical unknown nonlinear dynamics in networks with jointly connected topologies following a leader with also unknown nonlinear velocity dynamics. By parameterizing the unknown nonlinear dynamics of all agents by some basis functions, each agent estimating the unknown parameters, a decentralized adaptive consensus algorithm is developed in networks with jointly connected topologies by using both relative position feedback and local consensus error feedback of neighboring agents. By introducing Persistent excitation (PE) condition for regressor matrix, both position errors and parameter estimate errors can be proved to be globally uniformly asymptotically convergent to zero based on algebraic graph theory and Lyapunov theory.

The contributions of this paper are mainly in two aspects. Firstly, a novel type decentralized adaptive consensus control scheme is proposed for the considered multiagent systems to follow a leader in networks with jointly connected topologies, by relative position and local consensus error feedback. When unknown information or unmeasured information exists in the system, there are few efforts in the literature considering networks with switching topology, especially jointly connected topologies. Except for Hong et al. (2006), the works (Bai et al., 2008, 2009; Hou et al., 2009; Das and Lewis, 2010; Yu et al., 2009a,b) mentioned above are all for networks with fixed topology. In Hong et al. (2006), the case of networks with switching topologies and an extended case are studied. However, the algorithm proposed in Hong et al. (2006) is not strictly decentralized because each agent in the group must have access to the information $a_{0}(t)$ of the leader. Moreover, in the extended case of networks with switching topologies, it requires that each time interval has identical length, and the total period of connected interconnected graphs is sufficiently large. In our case, only jointly connectedness is assumed. Secondly, sufficient conditions are obtained for ensuring consensus with global, uniform and asymptotical parameter convergence. The consensus of all agents is ensured due to joint connectedness of graphs in networks with jointly connected topologies. The PE condition and some boundedness assumptions are introduced for ensuring parameter convergence. The parameter convergence analysis is more challenging when the interaction topology is switching. This is particularly true for the case of networks with jointly connected topologies, because standard results from adaptive control theory cannot be applied to the system directly. The two papers (Bai et al., 2008, 2009) also introduced PE condition for parameter convergence analysis in fixed network topology. A situation of all followers and the leader having non-identical unknown nonlinear dynamics and external disturbances is considered in Das and Lewis (2010), in which all consensus errors and parameter estimate errors are proved to be uniformly ultimately bounded (UUB) based on some assumptions in fixed network topologies. In $\mathrm{Yu}$ et al. (2009b), parameter convergence is not considered. In our work, both consensus errors and parameter estimate errors converging to zero (globally uniformly asymptotically) are obtained for switching networks with joint connectedness.

This paper is organized as follows. In section 2, we establish the notation and formally state the problem. We present our main results in section 3 , the simulation results supporting the objectives of the paper in section 4 and the concluding remarks in section 5 .

\section{Problem Statement}

We consider a multi-agent system consisting of $N$ agents and a leader. The dynamics of $N$ agents are described by

$$
\dot{x}_{i}(t)=f_{i}\left(x_{i}, t\right)+u_{i}(t), i=1,2, \cdots, N,
$$

where $x_{i}(t) \in R$ is the position state of $i$ th agent, $u_{i}(t) \in$ $R$ is the control input, and $f_{i}\left(x_{i}, t\right)$ is the dynamics of agent $i$, which is assumed to be unknown. Standard assumptions for existence of unique solutions are made, i.e., $f_{i}\left(x_{i}, t\right)$ is continuous in $t$ and Lipschitz in $x_{i}$. We assume that the leader agent moves in $R$ and its underlying dynamics is described by

$$
\dot{x}_{0}(t)=v_{0}(t)
$$

where $x_{0}(t) \in R$ is the position state of the leader, $v_{0}(t) \in R$ is its velocity and assumed to be unknown. The leader agent moves freely or along some planning trajectory, however, we assume that its velocity dynamics $v_{0}(t)$ is only related to time $t$ and unknown.

Remark 1 To avoiding complicated expressions, the states of all agents are assumed to be scalars in $R$, which is trivial to be extended to $R^{n}$ by introducing Kronecker product. Kronecker product of matrix $A \in R^{m \times n}$ and 
$B \in R^{p \times q}$ is defined as

$$
A \otimes B=\left(\begin{array}{ccc}
a_{11} B & \cdots & a_{1 n} B \\
\vdots & \ddots & \vdots \\
a_{m 1} B & \cdots & a_{m n} B
\end{array}\right) .
$$

The information exchange between agents in a multiagent system can be modeled using graphs. A graph $\mathcal{G}(\mathcal{V}, \mathcal{E})$ consists of a node set $\mathcal{V}=\{1,2, \cdots, N\}$ and an edge set $\mathcal{E} \subset \mathcal{V} \times \mathcal{V}$, where an edge of edge set $\mathcal{E}$ is denoted by $(i, j)$. A graph is undirected if edges $(i, j) \in \mathcal{E}$ are unordered pair. A graph is simple if it has no selfloops or repeated edges. If there is an edge between two nodes, then the two nodes are neighbors (or adjacent) to each other. The set of neighbors of node $i$ is denoted by $\mathcal{N}_{i}=\{j \in \mathcal{V} \mid(i, j) \in \mathcal{E}, j \neq i\}$. A path is a sequence of connected edges in a graph. If there is a path between any two nodes of a graph $\mathcal{G}$, then $\mathcal{G}$ is said to be connected, otherwise disconnected. The union of a collection of graphs is a graph with node set and edge set being the union of node set and edge set of all of the graphs in the collection. We say that a collection of graphs is jointly connected if the union of its members is connected.

With regarding the $N$ agents as the nodes in $\mathcal{V}$, the relationships between $N$ agents can be conveniently described by a simple and undirected graph $\mathcal{G}$, in which an undirected edge $(i, j)$ denotes that agent $i$ and $j$ can sense, receive or obtain information from each other. The adjacency matrix of graph $\mathcal{G}$ is denoted by $A=\left[a_{i j}\right] \in$ $R^{N \times N}$, whose $(i j)$ th entry is 1 if $(i, j)$ is an edge of graph $\mathcal{G}$ and 0 if it is not. The degree matrix $D \in R^{N \times N}$ of graph $\mathcal{G}$ is a diagonal matrix with $i$ th diagonal element being $\left|\mathcal{N}_{i}\right|$. The Laplacian of graph $\mathcal{G}$ is defined as $L=D-A$, which is symmetric and have following well-known results in algbraic graph theory (Godsil and Royle, 2001).

Lemma 2 Laplacian L of graph $\mathcal{G}$ has at least one zero eigenvalue with $\mathbf{1}_{N}=(1,1, \cdots, 1)^{T} \in R^{N}$ as its eigenvector, and all the non-zero eigenvalues of $L$ are positive. Laplacian $L$ has a simple zero eigenvalue if and only if graph $\mathcal{G}$ is connected.

To describe the information transmission between $N$ agents and the leader, we need define another graph $\overline{\mathcal{G}}$ on nodes $0,1,2, \cdots, N$, which consists of graph $\mathcal{G}$, node 0 representing the leader and edges between the leader and its neighbors.

Note that the interconnected topologies of the considered multi-agent system can vary with time. We need to consider all possible graphs $\left\{\overline{\mathcal{G}}_{p} \mid p \in \mathcal{P}\right\}$ on nodes set $\{0,1,2, \cdots, N\}$, where $\mathcal{P}$ is an index set. The subgraphs defined on node set $\{1,2, \cdots, N\}$ can denot- ed as $\left\{\mathcal{G}_{p} \mid p \in \mathcal{P}\right\}$ accordingly. To describe the dependence of graphs upon time, we define a switching signal $\sigma(t):[0, \infty) \rightarrow \mathcal{P}$, which is piecewise constant. Therefore, the underlying graphs at time $t$ on $N+1$ and $N$ nodes are denoted as $\overline{\mathcal{G}}_{\sigma(t)}$ and $\mathcal{G}_{\sigma(t)}$, respectively. The index number between agent $i$ and the leader is denoted by $b_{i}(t)$, which is defined to be 1 whenever leader agent is agent $i$ 's neighbor and 0 otherwise. Note that neighbors set $\mathcal{N}_{i}$ of all agent, all $(i j)$ th entries $a_{i j}$ of adjacency matrix $A$, and graph Laplacian $L$ are all time varying. We use $\mathcal{N}_{i}(t), a_{i j}(t)$ and $L_{\sigma(t)}$ to denote their time varying versions, respectively. It is assumed in this paper that $\sigma(t)$ switches finite times in any bounded time interval.

In this work, we consider the problem of designing decentralized controllers $u_{i}, i=1,2, \cdots, N$, such that all agents follow the leader.

For the multi-agent system (1)-(2), we say consensus is achieved if, for each agent $i \in\{1,2, \cdots, N\}$, there exists a controller $u_{i}$ such that

$$
\lim _{t \rightarrow \infty}\left|x_{i}(t)-x_{0}(t)\right|=0, i=1,2, \cdots, N
$$

for any initial condition $x_{i}(0), i=0,1, \cdots, N$.

When $v_{0}(t)$ is known and $f_{i}\left(x_{i}, t\right)=0, i=1,2, \cdots, N$, all agent can reach consensus on the time-varying leader, for example, using following control scheme (Ren, 2007):

$$
u_{i}=v_{0}(t)-\sum_{j \in \mathcal{N}_{i}} a_{i j}\left(x_{i}-x_{j}\right)-b_{i}\left(x_{i}-x_{0}\right) .
$$

When $v_{0}(t)$ and $f_{i}\left(x_{i}, t\right), i=1,2, \cdots, N$, are all unknown, it is a challenging work for all agents to achieve consensus.

\section{Main results}

In this section, we firstly give a basis function expression of the unknown nonlinear dynamics and the unknown velocity dynamics of the leader, and then a decentralized adaptive consensus algorithm is proposed in networks with switching topologies through relative position and local consensus error feedback. Finally, stability analysis of the consensus algorithm is given in switching networks with joint connectedness.

Suppose that the unknown nonlinear dynamics $f_{i}\left(x_{i}, t\right)$, $i=1, \cdots, N$, unknown velocity $v_{0}(t)$ of the leader, are parameterized as

$$
f_{i}\left(x_{i}, t\right)=\phi_{i}^{T}\left(x_{i}, t\right) \theta_{i}, i=1,2, \cdots, N
$$

and

$$
v_{0}(t)=\phi_{0}^{T}(t) \theta_{0},
$$


where $\phi_{0}(t), \phi_{i}\left(x_{i}, t\right) \in R^{m}$ are basis function column vectors and $\theta_{0}, \theta_{i} \in R^{m}$ are unknown constant parameter column vectors to be estimated.

Because $\theta_{0}$ is unavailable to each agent, the $i$ th agent estimates the unknown parameter vector $\theta_{0}$ by $\hat{\theta}_{0 i}$ and $v_{0}(t)$ by $\hat{v}_{0 i}(t)$ respectively. We have

$$
\hat{v}_{0 i}(t)=\phi_{0}^{T}(t) \hat{\theta}_{0 i}, i=1,2, \cdots, N
$$

Similarly, the estimate of $f_{i}\left(x_{i}, t\right)$ is expressed as

$$
\hat{f}_{i}\left(x_{i}, t\right)=\phi_{i}^{T}\left(x_{i}, t\right) \hat{\theta}_{i}, i=1,2, \cdots, N
$$

where $\hat{\theta}_{i}$ is the estimate of $\theta_{i}$.

Remark 3 The unknown nonlinear dynamics of all agents are assumed to be linearly parameterized. The linearly parameterized models have been studied widely in classical adaptive control (Sastry and Bodson, 1989; Marino and Tomei, 1995). The examples of linearly parameterized model of multi-agent systems can be found in Bai et al. (2008, 2009).

\subsection{Decentralized adaptive consensus algorithm design}

Consider an infinite sequence of bounded, nonoverlapping, contiguous time intervals $\left[t_{k}, t_{k+1}\right), k=$ $0,1,2, \cdots$, with $t_{0}=0, T_{0} \leq t_{k+1}-t_{k} \leq T$ for some positive constants $T_{0}, T$. Suppose that in each time interval $\left[t_{k}, t_{k+1}\right)$ there exists a sequence of nonoverlapping, contiguous subinterval

$$
\left[t_{k}^{0}, t_{k}^{1}\right), \ldots,\left[t_{k}^{l}, t_{k}^{l+1}\right), \cdots,\left[t_{k}^{l_{k}-1}, t_{k}^{l_{k}}\right)
$$

with $t_{k}=t_{k}^{0}, t_{k+1}=t_{k}^{l_{k}}$ for some integer $l_{k} \geq 0$ such that the time-varying graph topology switches at time instants $t_{k}^{0}, t_{k}^{1}, \cdots, t_{k}^{l_{k}-1}$, in other words, the switching graph $\overline{\mathcal{G}}_{\sigma(t)}$ is time invariant in each of such subintervals. We assume that there exists a constant number $\tau>0$, often called dwell time, such that $t_{k}^{l+1}-t_{k}^{l} \geq \tau, 0 \leq l \leq$ $l_{k}-1$. Note that in each of such subintervals the interconnected graph $\overline{\mathcal{G}}_{\sigma(t)}$ is permitted to be disconnected. A collection of switching graphs $\left\{\overline{\mathcal{G}}_{\sigma(s)} \mid s \in[t, t+\Delta\right.$ $t], \Delta t>0\}$ is said to be jointly connected across a time interval $[t, t+\Delta t]$ if its union is connected. For each $p \in \mathcal{P}, H_{p}$ has $N$ eigenvalues denoted as $\lambda_{p}^{1}, \lambda_{p}^{2}, \cdots, \lambda_{p}^{N}$ based on some labeling rule (Ni and Cheng, 2010). Define $\mathscr{C}(p)=\left\{k \mid \lambda_{p}^{k} \neq 0, k=1,2, \cdots, N\right\}$, we have the following Lemma (Ni and Cheng, 2010):

Lemma 4 Graphs $\mathcal{G}_{p}, p \in \mathcal{P}$ are jointly connected across $\left[t_{k}, t_{k+1}\right)$ if and only if $\bigcup_{t \in\left[t_{k}, t_{k+1}\right)} \mathscr{C}(\sigma(t))=$ $\{1,2, \cdots, N\}$.
Define local consensus error vector (Khoo et al., 2009) for agent $i$ as

$$
\begin{aligned}
e_{i}(t)= & \sum_{j \in \mathcal{N}_{i}(t)} a_{i j}(t)\left(x_{i}-x_{j}\right) \\
& +b_{i}(t)\left(x_{i}-x_{0}\right), i=1, \cdots, N .
\end{aligned}
$$

The global error vector for switching graph $\overline{\mathcal{G}}_{\sigma(t)}$ is expressed as

$$
e(t)=H_{\sigma(t)}\left(x-\mathbf{1}_{N} x_{0}\right),
$$

where $H_{\sigma(t)}=L_{\sigma(t)}+B_{\sigma(t)}, L_{\sigma(t)}$ is the Laplacian of graph $\mathcal{G}_{\sigma(t)}$ and $B_{\sigma(t)}$ is a diagonal matrix with diagonal elements $b_{1}(t), b_{2}(t), \cdots, b_{N}(t)$.

In each time interval $\left[t_{k}^{l}, t_{k}^{l+1}\right), k=0,1, \cdots, 0 \leq l \leq$ $l_{k}-1$, matrix $H_{\sigma(t)}$ is time-invariant, then

$$
\begin{aligned}
\dot{e}(t) & =H_{\sigma(t)}\left(\dot{x}-\mathbf{1}_{N} \dot{x}_{0}\right) \\
& =H_{\sigma(t)}\left(f+u-\mathbf{1}_{N} v_{0}(t)\right)
\end{aligned}
$$

where $f=\left(f_{1}, f_{2}, \cdots, f_{N}\right)^{T}$.

We denote by $\operatorname{col}\left(x_{i}\right)_{i \in S}$ or $\operatorname{col}\left(x_{i}\right)$ according to the context the stack column vector of $x_{i}$ with $i$ in some index set $S$, by $\operatorname{col}(x, y)$ the stack column vector of vector $x$ and $y$, etc. Letting $x=\operatorname{col}\left(x_{i}\right), \bar{x}=x-\mathbf{1}_{N} x_{0}, u=\operatorname{col}\left(u_{i}\right)$, $\hat{\Theta}_{0}=\operatorname{col}\left(\hat{\theta}_{0 i}\right), \hat{\Theta}_{f}=\operatorname{col}\left(\hat{\theta}_{i}\right)$ with $i \in\{1,2, \cdots, N\}$, and $\sigma(t):[0, \infty) \rightarrow \mathcal{P}$ a switching signal, we propose the following adaptive consensus scheme consisting of two parts.

Decentralized feedback laws:

$$
\begin{aligned}
u_{i}(t)= & c \sum_{j \in \mathcal{N}_{i}(t)} a_{i j}(t)\left(x_{j}-x_{i}\right) \\
& +c b_{i}(t)\left(x_{0}-x_{i}\right)+\phi_{0}^{T}(t) \hat{\theta}_{0 i} \\
& -\phi_{i}\left(x_{i}, t\right)^{T} \hat{\theta}_{i}, i=1,2, \cdots, N,
\end{aligned}
$$

or in matrix form

$$
u=-c H_{\sigma(t)} \bar{x}+\Phi_{0}^{T} \hat{\Theta}_{0}-\Phi_{f}^{T} \hat{\Theta}_{f} .
$$

where $c>0$ is a constant number, $\Phi_{0}=\left(I_{N} \otimes \phi_{0}\right), \Phi_{f}$ is a block diagonal matrix with block diagonal elements $\phi_{1}, \phi_{2}, \cdots, \phi_{N}, I_{N}$ is the $N \times N$ identity matrix.

Decentralized adaptive laws:

$$
\begin{aligned}
& \dot{\hat{\theta}}_{0 i}=-\frac{c_{0}}{c} \phi_{0}(t)\left[\sum_{j \in \mathcal{N}_{i}(t)} a_{i j}(t)\left(e_{i}-e_{j}\right)+b_{i}(t) e_{i}\right], \\
& \dot{\hat{\theta}}_{i}=\frac{c_{1}}{c} \phi_{i}\left(x_{i}, t\right)\left[\sum_{j \in \mathcal{N}_{i}(t)} a_{i j}(t)\left(e_{i}-e_{j}\right)+b_{i}(t) e_{i}\right], \\
& i=1,2, \cdots, N
\end{aligned}
$$


or in matrix form

$$
\begin{aligned}
& \dot{\hat{\Theta}}_{0}=-\frac{c_{0}}{c} \Phi_{0} H_{\sigma(t)} e, \\
& \dot{\hat{\Theta}}_{f}=\frac{c_{1}}{c} \Phi_{f} H_{\sigma(t)} e .
\end{aligned}
$$

where $c_{0}, c_{1}>0$ are constant numbers.

Remark 5 Note that controller $u_{i}(t)$ defined in (13) is decentralized. For control purpose, we assume that the information of local consensus error $e_{i}(t)$ of agent $i$ is calculated and saved in its memory at each time instant by each agent and available for its neighbors. Controller $u_{i}(t)$ only depends on the information of relative position measurements and local consensus errors from its neighboring agents. Similar way of information transmission can be found, for instance, in the literature (Ren, 2007; Ren et al., 2009; Khoo et al., 2009).

Remark 6 If the states $x_{i}(t), i=0,1, \cdots, N$, of system (1)-(2) are considered in $R^{n}$, a corresponding version of equation (14) and (16) are the following equations

$$
\begin{aligned}
u & =-c e+\left(\Phi_{0}^{T} \otimes I_{n}\right) \hat{\Theta}_{0}-\left(\Phi_{f}^{T} \otimes I_{n}\right) \hat{\Theta}_{f} \\
\dot{\hat{\Theta}}_{0} & =-\frac{c_{0}}{c}\left(\Phi_{0} H_{\sigma(t)} \otimes I_{n}\right) e \\
\dot{\hat{\Theta}}_{f} & =\frac{c_{1}}{c}\left(\Phi_{f} H_{\sigma(t)} \otimes I_{n}\right) e .
\end{aligned}
$$

where $e=\left(H_{\sigma(t)} \otimes I_{n}\right)\left(x-\mathbf{1}_{N} \otimes x_{0}\right)$.

\subsection{Stability analysis}

Denote $\Theta_{0}=\mathbf{1}_{N} \otimes \theta_{0}, \bar{\theta}_{0 i}=\hat{\theta}_{0 i}-\theta_{0}, \bar{\Theta}_{0}=\hat{\Theta}_{0}-\Theta_{0}=$ $\operatorname{col}\left(\bar{\theta}_{0 i}\right), \Theta_{f}=\operatorname{col}\left(\theta_{i}\right), \bar{\theta}_{i}=\hat{\theta}_{i}-\theta_{i}, \bar{\Theta}_{f}=\hat{\Theta}_{f}-\Theta_{f}=$ $\operatorname{col}\left(\bar{\theta}_{i}\right)$, and $\bar{\Theta}=\operatorname{col}\left(\bar{\Theta}_{0}, \bar{\Theta}_{f}\right)$. From equations (10)-(16), we obtain the following error dynamics of the system (1)-(2):

$$
\left\{\begin{aligned}
\dot{e} & =-c H_{\sigma(t)} e+H_{\sigma(t)} \Phi_{0}^{T} \bar{\Theta}_{0}-H_{\sigma(t)} \Phi_{f}^{T} \bar{\Theta}_{f} \\
\dot{\bar{\Theta}}_{0} & =-\frac{c_{0}}{c} \Phi_{0} H_{\sigma(t)} e \\
\dot{\Theta}_{f} & =\frac{c_{1}}{c} \Phi_{f} H_{\sigma(t)} e .
\end{aligned}\right.
$$

The matrix $H_{\sigma(t)}$ corresponding to a graph $\overline{\mathcal{G}}_{\sigma(t)}$, has the following well-known properties (Ni and Cheng, 2010):

Lemma 7 (1) Matrix $H_{\sigma(t)}$ has nonnegative eigenvalues; (2) Matrix $H_{\sigma(t)}$ is positive definite if and only if graph $\overline{\mathcal{G}}_{\sigma(t)}$ is connected.

Let $P$ be a positive semi-definite matrix , and $\lambda_{\min }(P)$, $\lambda_{\max }(P)$ denote the smallest and the largest non-zero eigenvalue of matrix $P$ respectively. For each $p \in \mathcal{P}$, define $\mu_{p}=\lambda_{\min }\left(H_{p}\right)$ and $\nu_{p}=\lambda_{\max }\left(H_{p}\right)$. Based on Lemma 7 and the fact that the index set $\mathcal{P}$ is finite,

$$
\delta_{\min }=\min \left\{\mu_{p} \mid p \in \mathcal{P}\right\}, \delta_{\max }=\max \left\{\nu_{p} \mid p \in \mathcal{P}\right\}
$$

are positive and independent of time $t$.

Before giving the theoretical results, we suppose that the regressor matrix $\Phi=\left(\begin{array}{c}\Phi_{0} \\ \Phi_{f}\end{array}\right)$, is persistently exciting (PE) (Marino and Tomei, 1995), that is, there exist two positive reals $\delta_{0}$ and $\alpha$, such that

$$
\int_{t}^{t+\delta_{0}} \Phi \Phi^{T} d \tau \geq \alpha I>0, \forall t \geq 0
$$

This PE condition ensures the information richness of the time varying matrix $\Phi$ throughout time, and guarantees parameter convergence, i.e.,

$$
\lim _{t \rightarrow \infty}\left\|\hat{\theta}_{0 i}-\theta_{0}\right\|=0, \lim _{t \rightarrow \infty}\left\|\hat{\theta}_{i}-\theta_{i}\right\|=0
$$

for any initial condition $\hat{\theta}_{0 i}(0), \hat{\theta}_{i}(0), i=1,2, \cdots, N$.

The PE condition has another interpretation, by reexpressing the $\mathrm{PE}$ condition in scalar form

$$
\int_{t}^{t+\delta_{0}} \omega^{T} \Phi \Phi^{T} \omega d \tau \geq \alpha, \forall t \geq 0, \forall \omega:\|\omega\|=1 .
$$

Lemma 8 If graphs $\mathcal{G}_{p}, p \in \mathcal{P}$ are jointly connected across each time interval $\left[t_{k}, t_{k+1}\right)$, then $\lim _{t \rightarrow \infty} e(t)=0$ implies $\lim _{t \rightarrow \infty} \bar{x}(t)=0$.

PROOF. Due to the symmetry of $H_{p}$, there exists an orthogonal matrix $U_{p}$ such that

$$
U_{p} H_{p} U_{p}^{T}=\Lambda_{p}=\operatorname{diag}\left\{\lambda_{p}^{i_{1}}, \lambda_{p}^{i_{2}}, \cdots, \lambda_{p}^{i_{N}}\right\}
$$

where $\lambda_{p}^{i_{1}}, \lambda_{p}^{i_{2}}, \cdots, \lambda_{p}^{i_{N}}$ are the $N$ eigenvalues of $H_{p}$, $i_{1}, i_{2}, \cdots, i_{N}$ form a permutation of $1,2, \cdots, N$.

Let $\varepsilon=U_{p} \bar{x}$, we have

$$
\bar{x}^{T} e=\bar{x}^{T} H_{p} \bar{x}=\varepsilon^{T} \Lambda_{p} \varepsilon \geq \delta_{\min } \sum_{i \in \mathscr{C}\left(\sigma\left(t_{k}^{l}\right)\right)} \varepsilon_{i}^{2} \geq 0
$$

for $l=0,1, \cdots, l_{k}-1$.

Due to the jointly connectedness of $\mathcal{G}_{p}, \lim _{t \rightarrow \infty} e(t)=0$ and Lemma 4, we have $\lim _{t \rightarrow \infty} \sum_{l=0}^{l_{k}-1} \sum_{i \in \mathscr{C}\left(\sigma\left(t_{k}^{l}\right)\right)} \varepsilon_{i}^{2}=$ $\lim _{t \rightarrow \infty} \sum_{i=1}^{N} a_{i} \varepsilon_{i}^{2}=0$, where $a_{1}, a_{2}, \cdots, a_{N}$ are some 
positive integers. This implies $\lim _{t \rightarrow \infty} \varepsilon_{i}=0, i=$ $1,2, \cdots, N$. Then $\lim _{t \rightarrow \infty} \bar{x}=0$.

The following theorem is our main result:

Theorem 9 Consider the multi-agent system (1)-(2). Assume that the switching interconnected graph $\overline{\mathcal{G}}_{\sigma(t)}$ is jointly connected across each time interval $\left[t_{k}, t_{k+1}\right), k=$ $0,1, \cdots, \phi_{i}$ and $\dot{\phi}_{i}, i=0,1, \cdots, N$, are uniformly bounded and the PE condition defined in (19) is satisfied, then, under control law (13) and parameter adaptive law (15), $(\bar{x}, \bar{\Theta})=0$ is a globally uniformly asymptotically stable equilibrium point, i.e., consensus is reached with global, uniform and asymptotical parameter convergence.

PROOF. For multi-agent system (17), consider a Lyapunov function candidate

$$
V(t)=\frac{1}{2 c} e^{T} e+\frac{1}{2 c_{0}} \bar{\Theta}_{0}^{T} \bar{\Theta}_{0}+\frac{1}{2 c_{1}} \bar{\Theta}_{f}^{T} \bar{\Theta}_{f} .
$$

Obviously, $V(t)$ is continuously differentiable at any time except for the switching instants.

At a non-switching time $t$, assume that the subsystem $p \in \mathcal{P}$ is active, the time derivative of this Lyapunov function candidate along the trajectory of the system $(17)$ is $\dot{V}(t)=-e^{T} H_{p} e$. From (22), let $\tilde{e}=U_{p} e$, we have

$$
\begin{aligned}
\dot{V}(t) & =-\tilde{e}^{T} \Lambda_{p} \tilde{e} \\
& =-\sum_{i \in \mathscr{C}(p)} \lambda_{p}^{i} \tilde{e}_{i}^{T} \tilde{e}_{i} \\
& \leq-\delta_{p} \sum_{i \in \mathscr{C}(p)} \tilde{e}_{i}^{T} \tilde{e}_{i} \\
& \leq-\delta_{\min } \sum_{i \in \mathscr{C}(p)} \tilde{e}_{i}^{T} \tilde{e}_{i} \\
& \leq 0 .
\end{aligned}
$$

Therefore, $\lim _{t \rightarrow \infty} V(t)=V(\infty)$ exists.

In the following, we prove $\lim _{t \rightarrow \infty} e(t)=0$.

Consider the infinite sequence $\left\{V\left(t_{k}\right), k=0,1, \cdots\right\}$ and from Cauchy's convergence criteria, we have that, for $\forall \epsilon>0$, there exists a positive integer $K$, such that, for $\forall k>K,\left|V\left(t_{k+1}\right)-V\left(t_{k}\right)\right|<\epsilon$ or equivalently, $\left|\int_{t_{k}}^{t_{k+1}} \dot{V}(t) d t\right|<\epsilon$. This integral can be rewritten as $\sum_{l=0}^{l_{k}-1} \int_{t_{k}^{l}}^{t_{k}^{l+1}} \dot{V}(t) d t>-\epsilon$.

From (24), we have

$$
\begin{aligned}
-\epsilon & <\sum_{l=0}^{l_{k}-1} \int_{t_{k}^{l}}^{t_{k}^{l+1}} \dot{V}(t) d t \\
& \leq-\delta_{\min } \sum_{l=0}^{l_{k}-1} \int_{t_{k}^{l}}^{t_{k}^{l+1}} \sum_{i \in \mathscr{C}\left(\sigma\left(t_{k}^{l}\right)\right)} \tilde{e}_{i}^{T} \tilde{e}_{i} d t
\end{aligned}
$$

and

$$
\begin{aligned}
& \sum_{l=0}^{l_{k}-1} \int_{t_{k}^{l}}^{t_{k}^{l}+\tau} \sum_{i \in \mathscr{C}\left(\sigma\left(t_{k}^{l}\right)\right)} \tilde{e}_{i}^{T} \tilde{e}_{i} d t \\
& \leq \sum_{l=0}^{l_{k}-1} \int_{t_{k}^{l}}^{t_{k}^{l+1}} \sum_{i \in \mathscr{C}\left(\sigma\left(t_{k}^{l}\right)\right)} \tilde{e}_{i}^{T} \tilde{e}_{i} d t \leq \frac{\epsilon}{\delta_{\min }} .
\end{aligned}
$$

Because $l_{k}$ is assumed to be finite in each time interval $\left[t_{k}, t_{k+1}\right)$ for $k=0,1, \cdots$. Thus, for $\forall k>K$, we have

$$
\int_{t_{k}^{l}}^{t_{k}^{l}+\tau} \sum_{i \in \mathscr{C}\left(\sigma\left(t_{k}^{l}\right)\right)} \tilde{e}_{i}^{T} \tilde{e}_{i} d t \leq \frac{\epsilon}{\delta_{\min }}, l=0,1, \cdots, l_{k}-1
$$

or equivalently,

$$
\lim _{t \rightarrow \infty} \int_{t}^{t+\tau} \sum_{i \in \mathscr{C}\left(\sigma\left(t_{k}^{l}\right)\right)} \tilde{e}_{i}^{T}(s) \tilde{e}_{i}(s) d s=0
$$

which implies that

$$
\lim _{t \rightarrow \infty} \sum_{l=0}^{l_{k}-1} \int_{t}^{t+\tau} \sum_{i \in \mathscr{C}\left(\sigma\left(t_{k}^{l}\right)\right)} \tilde{e}_{i}^{T}(s) \tilde{e}_{i}(s) d s=0 .
$$

From Lemma $4, \bigcup_{t \in\left[t_{k}, t_{k+1}\right)} \mathscr{C}(\sigma(t))=\{1,2, \cdots, N\}$ due to the joint connectivity of the graphs through the time interval $\left[t_{k}, t_{k+1}\right)$, we have

$$
\lim _{t \rightarrow \infty} \int_{t}^{t+\tau} \sum_{i=1}^{N} a_{i} \tilde{e}_{i}^{T}(s) \tilde{e}_{i}(s) d s=0
$$

where $a_{i}, i=1,2, \cdots, N$, are some positive integers. Moreover, from (23) and (24) it follows that both $e$ and $\bar{\Theta}$ are uniformly bounded for any $t \geq 0$ and so is $\dot{e}$ due to (17) and the assumption that $\phi_{i}$ and $\dot{\phi}_{i}, i=0,1, \cdots, N$, are uniformly bounded. Therefore, $\sum_{i=1}^{N} a_{i} \tilde{e}_{i}^{T}(s) \tilde{e}_{i}(s) d s$ is uniformly continuous. From Barbalat's Lemma, we have $\lim _{t \rightarrow \infty} \sum_{i=1}^{N} a_{i} \tilde{e}_{i}^{T}(t) \tilde{e}_{i}(t) d t=$ 0 , then $\lim _{t \rightarrow \infty} \tilde{e}_{i}(t)=0, i=1,2, \cdots, N$. Thus $\lim _{t \rightarrow \infty} e_{i}(t)=0, i=1,2, \cdots, N$. Therefore, from Lem$\operatorname{ma} 8, \lim _{t \rightarrow \infty}\left|x_{i}(t)-x_{0}(t)\right|=0, i=1,2, \cdots, N$.

Now, to show that for any initial condition

$$
\lim _{t \rightarrow \infty}\|\bar{\Theta}(t)\|=0
$$

i.e., for any $\epsilon>0$ there exists $\mathcal{T}_{\epsilon}>0$ such that $\|\bar{\Theta}(t)\|<$ $\epsilon, \forall t>\mathcal{T}_{\epsilon}$. We first prove the following claim.

Claim 10 Given any $\epsilon>0$ and $\mathcal{T}>0$, for any initial condition $e(0), \bar{\Theta}(0)$ there exists $t>\mathcal{T}$ such that $\left\|\operatorname{col}\left(\bar{\theta}_{0 i}, \bar{\theta}_{i}\right)\right\|<\epsilon, i=1,2, \cdots, N$. 
Proof of Claim 10. We equivalently show by contradiction that for any $\epsilon>0$ and some $i \in\{1,2, \cdots, N\}$ a time $\mathcal{T}_{1}$ such that

$$
\left\|\operatorname{col}\left(\bar{\theta}_{0 i}, \bar{\theta}_{i}\right)\right\|>\epsilon, \forall t \geq \mathcal{T}_{1}
$$

does not exist.

Without loss of generality, for the infinite sequence of time intervals $\left[t_{k}, t_{k+1}\right), k=0,1, \cdots$, consider an infinite subsequence of time intervals $\left[t_{k_{j}}, t_{k_{j}+1}\right), j=0,1, \cdots$ with identical length $T_{1}$ satisfying $T_{0} \leq T_{1} \leq T$, that is $t_{k_{j}+1}=t_{k_{j}}+T_{1}$. Define function

$$
\Psi(\bar{\Theta}(t), t)=\frac{1}{2}\left[\bar{\Theta}^{T}\left(t+T_{1}\right) \bar{\Theta}\left(t+T_{1}\right)-\bar{\Theta}^{T}(t) \bar{\Theta}(t)\right] .
$$

Because $\lim _{t \rightarrow \infty} e(t)=0, \lim _{t \rightarrow \infty} V(t)=V(\infty)$ exists and (23), we have $\lim _{t \rightarrow \infty} \vec{\Theta}(t)^{T} \vec{\Theta}(t)=\eta V(\infty)$ with $\eta$ some positive constant number and then $\lim _{t \rightarrow \infty} \Psi(\bar{\Theta}(t), t)=0$ due to (31). Therefor, for $\forall \epsilon_{1}>0$, there exists $t_{\epsilon_{1}}>0$ such that

$$
\left\|\Psi(\bar{\Theta}(t), t)-\Psi\left(\bar{\Theta}\left(t^{\prime}\right), t^{\prime}\right)\right\|<\epsilon_{1}, \forall t, t^{\prime}>t_{\epsilon_{1}} .
$$

The time derivative of the function $\Psi(\bar{\Theta}(t), t)$ defined in (31) at time instant $t_{k_{j}}$ is

$$
\begin{aligned}
& \dot{\Psi}\left(\bar{\Theta}\left(t_{k_{j}}\right), t_{k_{j}}\right) \\
= & \int_{t_{k_{j}}}^{t_{k_{j}}+T_{1}} \frac{d}{d \tau}\left[\bar{\Theta}^{T}(\tau) \dot{\bar{\Theta}}(\tau)\right] d \tau \\
= & -\frac{1}{c} \sum_{l=0}^{l_{k}-1} \int_{t_{k_{j}}^{t_{k}}}^{t_{k_{j}}^{l+1}} \frac{d}{d \tau}\left\{\left[c_{0} \bar{\Theta}_{0}^{T} \Phi_{0}-c_{1} \bar{\Theta}_{f}^{T} \Phi_{f}\right] H_{p} e\right\} d \tau \\
= & \sum_{l=0}^{l_{k}-1} \int_{t_{k_{j}}^{t_{k_{j}}}}^{t^{2}}\left[\left(\frac{c_{0}^{2}}{c^{2}} e^{T} H_{p} \Phi_{0}^{T} \Phi_{0}-\frac{c_{0}}{c} \bar{\Theta}_{0}^{T} \dot{\Phi}_{0}+\frac{c_{1}}{c} \bar{\Theta}_{f}^{T} \dot{\Phi}_{f}\right.\right. \\
& \left.\left.+\frac{c_{1}^{2}}{c^{2}} e^{T} H_{p} \Phi_{f}^{T} \Phi_{f}\right) H_{p}+\left(c_{0} \bar{\Theta}_{0}^{T} \Phi_{0}-c_{1} \bar{\Theta}_{f}^{T} \Phi_{f}\right) H_{p}^{2}\right] e d \tau \\
& -\sum_{l=0}^{l_{k}-1} \int_{t_{k_{j}}^{l}}^{t_{k_{j}}^{l+1}}\left(\frac{c_{0}}{c} \bar{\Theta}_{0}^{T},-\frac{c_{1}}{c} \bar{\Theta}_{f}^{T}\right) \Phi H_{p}^{2} \Phi^{T}\left(\begin{array}{c}
\bar{\Theta}_{0} \\
-\bar{\Theta}_{f}
\end{array}\right) d \tau \\
\triangleq & \mathcal{I}_{1}-\mathcal{I}_{2} .
\end{aligned}
$$

Because $e, \bar{\Theta}, \phi_{i}, \dot{\phi}_{i}$ are bounded, letting $M_{e}, M_{\Theta}, M_{\Phi}>$ 0 be such that $\|e\| \leq M_{e},\left\|\bar{\Theta}_{0}\right\| \leq M_{\Theta},\left\|\bar{\Theta}_{f}\right\| \leq M_{\Theta}$, $\left\|\Phi_{0}\right\| \leq M_{\Phi},\left\|\Phi_{f}\right\| \leq M_{\Phi},\left\|\dot{\Phi}_{0}\right\| \leq M_{\Phi}$ and $\left\|\dot{\Phi}_{f}\right\| \leq M_{\Phi}$, $\forall t \geq 0$, we can write for the first integral in (33) as

$$
\mathcal{I}_{1} \leq M \sum_{l=0}^{l_{k}-1} \int_{t_{k_{j}}^{l}}^{t_{k_{j}}^{l+1}}\|e\| d \tau
$$

where $M=\left[\frac{c_{0}^{2}+c_{1}^{2}}{c^{2}} \delta_{\max } M_{e}+\frac{\left(c_{0}+c_{1}\right)\left(1+c \delta_{\max }\right)}{c} M_{\Theta}\right] \delta_{\max } M_{\Phi}$ is a positive constant.
Because $l_{k}, k=1,2, \cdots$, are assumed to be finite and $\lim _{t \rightarrow \infty}\|e(t)\|=0$, we have

$$
\mathcal{I}_{1} \leq \frac{c_{0}}{2 c} \alpha \gamma_{\min } \delta_{\min }^{2} \epsilon^{2}, \forall t_{k_{j}} \geq \mathcal{T}_{2},
$$

where $\gamma_{\min }$ is defined in the sequel.

From (22), we have

$$
H_{p}^{2}=U_{p}^{T} \Lambda_{p}^{2} U_{p} \geq \gamma_{p} \Lambda_{p}^{2},
$$

for some $0<\gamma_{p} \leq 1$.

Without loss of generality, selecting $c_{1}=c_{0}$ and letting $\tilde{\Theta}=\operatorname{col}\left(\bar{\Theta}_{0},-\bar{\Theta}_{f}\right)$, we have

$$
\mathcal{I}_{2} \geq \frac{c_{0}}{c} \sum_{l=0}^{l_{k}-1} \int_{t_{k_{j}}^{l}}^{t_{k_{j}}^{l+1}} \gamma_{p} \tilde{\Theta}^{T} \Phi \Lambda_{p}^{2} \Phi^{T} \tilde{\Theta} d \tau
$$

Let $\gamma_{\min }=\min \left\{\gamma_{p}: p \in \mathcal{P}\right\},\left(\Phi^{T} \tilde{\Theta}\right)_{i}$ represent the $i$ th component of $\Phi^{T} \tilde{\Theta}, \operatorname{col}\left(\bar{\theta}_{0 i}\right)$ and $\operatorname{col}\left(\bar{\theta}_{i}\right)$ be column vectors with components indexed by $i \in \mathscr{C}\left(\sigma\left(t_{k_{j}}^{l}\right)\right), \tilde{\Theta}^{p}=$ $\operatorname{col}\left[\operatorname{col}\left(\bar{\theta}_{0 i}\right)_{i \in \mathscr{C}\left(\sigma\left(t_{k_{j}}^{l}\right)\right)}, \operatorname{col}\left(\bar{\theta}_{i}\right)_{i \in \mathscr{C}\left(\sigma\left(t_{k_{j}}^{l}\right)\right)}\right], M_{i, N+i}$ be the principle submatrix of $\Phi \Phi^{T}$ by retaining like-numbered rows and columns indexed by $\left\{i, N+i: i \in \mathscr{C}\left(\sigma\left(t_{k_{j}}^{l}\right)\right)\right\}$, we have

$$
\begin{aligned}
\mathcal{I}_{2} \geq & \frac{c_{0}}{c} \gamma_{\min } \delta_{\min }^{2} \sum_{l=0}^{l_{k}-1} \\
& \int_{t_{k_{j}}^{l}}^{l_{k_{j}}^{++1}} \sum_{i \in \mathscr{C}\left(\sigma\left(t_{k_{j}}^{l}\right)\right)}\left(\Phi^{T} \tilde{\Theta}\right)_{i}^{2} d \tau \\
= & \frac{c_{0}}{c} \gamma_{\min } \delta_{\min }^{2} \sum_{l=0}^{l_{k}-1} \\
& \int_{t_{k_{j}}^{l}}^{l_{k_{j}}^{++1}}\left(\tilde{\Theta}^{p}\right)^{T} M_{i, N+i} \tilde{\Theta}^{p} d \tau .
\end{aligned}
$$

From the PE condition (19), we have

$$
\int_{t}^{t+\delta_{0}} M_{i, N+i} d \tau \geq \alpha I>0, \forall t \geq 0 .
$$

Assume now by contradiction that exists a time $\mathcal{T}_{1}$ so that (30) holds, which implies that for some $p,\left\|\tilde{\Theta}^{p}\right\|>\epsilon$, due to Lemma 4. From (21), (38), (39) and for some $l$

$$
\begin{aligned}
\mathcal{I}_{2} & \geq \frac{c_{0}}{c} \gamma_{\min } \delta_{\min }^{2} \int_{t_{k_{j}}^{l}}^{t_{k_{j}}^{l}+\tau}\left(\tilde{\Theta}^{p}\right)^{T} M_{i, N+i} \tilde{\Theta}^{p} d s \\
& \geq \frac{c_{0}}{c} \gamma_{\min } \delta_{\min }^{2} \epsilon^{2} \int_{t_{k_{j}}^{l}}^{t_{k_{j}}^{l}+\tau} \frac{\left(\tilde{\Theta}^{p}\right)^{T}}{\left\|\tilde{\Theta}^{p}\right\|} M_{i, N+i} \frac{\tilde{\Theta}^{p}}{\left\|\tilde{\Theta}^{p}\right\|} d s \\
& \geq \frac{c_{0}}{c} \alpha \gamma_{\min } \delta_{\min }^{2} \epsilon^{2}, \forall t_{k_{j}} \geq \mathcal{T}_{1} .
\end{aligned}
$$


From (33), (35) and (40), we obtain

$$
\dot{\Psi}\left(\bar{\Theta}\left(t_{k_{j}}\right), t_{k_{j}}\right) \leq-\frac{c_{0}}{2 c} \alpha \gamma_{\min } \delta_{\min }^{2} \epsilon^{2}<0, \forall t_{k_{j}} \geq \mathcal{T}_{3}
$$

with $\mathcal{T}_{3}=\max \left\{\mathcal{T}_{1}, \mathcal{T}_{2}, t_{\epsilon_{1}}\right\}$. Thus, from sign-preserving theorem of continuous function, there exists an interval $\left[t_{k_{j}}, t_{k_{j}}+\delta\right)$ with $t_{k_{j}} \geq \mathcal{T}_{3}, \delta>0$ such that $\dot{\Psi}(\bar{\Theta}(t), t)<$ $-\frac{c_{0}}{4 c} \alpha \gamma_{\min } \delta_{\min }^{2} \epsilon^{2}<0$ holds for $\forall t \in\left[t_{k_{j}}, t_{k_{j}}+\delta\right)$. Integrating $\dot{\Psi}(\bar{\Theta}(t), t)$ from $t_{k_{j}}$ to $t_{k_{j}}+\delta$ and selecting $\epsilon_{1}=\frac{c_{0}}{4 c} \alpha \gamma_{\min } \delta_{\min }^{2} \epsilon^{2} \delta$, we have

$$
\Psi\left(\bar{\Theta}\left(t_{k_{j}}\right), t_{k_{j}}\right)-\Psi\left(\bar{\Theta}\left(t_{k_{j}}+\delta\right), t_{k_{j}}+\delta\right)>\epsilon_{1}
$$

which contradicts (32). This completes the proof of the Claim.

By virtual of $\lim _{t \rightarrow \infty} e(t)=0$, for any $\epsilon>0$ there exists a time instant $t_{\epsilon}$ such that

$$
\|e(t)\| \leq \sqrt{\frac{c}{2 \min \left\{c_{0}, c_{1}\right\}}} \epsilon, \forall t \geq t_{\epsilon} .
$$

By virtual the Claim there exists a time instant $\mathcal{T}_{\epsilon}>t_{\epsilon}$ such that

$$
\|\bar{\Theta}(t)\| \leq \frac{1}{\sqrt{2}} \epsilon .
$$

From the initial condition $e\left(\mathcal{T}_{\epsilon}\right)$ and $\bar{\Theta}\left(\mathcal{T}_{\epsilon}\right)$, according to (23), (43), and (44), we have

$$
\|\bar{\Theta}\| \leq \sqrt{\frac{\min \left\{c_{0}, c_{1}\right\}}{c}\left\|e\left(\mathcal{T}_{\epsilon}\right)\right\|^{2}+\left\|\bar{\Theta}\left(\mathcal{T}_{\epsilon}\right)\right\|^{2}} \leq \epsilon, \forall t \geq \mathcal{T}_{\epsilon}
$$

which implies (29). Therefore the equilibrium is attractive. Since $\lim _{t \rightarrow \infty} e(t)=0$ and $\lim _{t \rightarrow \infty} \bar{\Theta}(t)=$ 0 hold uniformly with respect to initial time instant, it follows that $(e, \bar{\Theta})$ is a globally uniformly asymptotically stable equilibrium point. Note that $\lim _{t \rightarrow \infty}\|e\|=0$ implies $\lim _{t \rightarrow \infty}\|\bar{x}\|=0$ due to Lemma 8, therefore $\lim _{t \rightarrow \infty}\left|x_{i}(t)-x_{0}(t)\right|=0$, for $\forall x_{i}(0) \in R, i=1,2, \cdots, N$ and $\lim _{t \rightarrow \infty}\left\|\hat{\theta}_{0 i}-\theta_{0}\right\|=0$, $\lim _{t \rightarrow \infty}\left\|\hat{\theta}_{i}-\theta_{i}\right\|=0$, for $\forall \hat{\theta}_{0 i}(0) \in R^{m}, \hat{\theta}_{i}(0) \in R^{m}, i=$ $1,2, \cdots, N$.

Remark 11 The consensus stability proof of Theorem 9, which uses some inequality techniques, Lemma 4 and Barbalat's Lemma, is motivated by that in Ni and Cheng (2010). The Lemma 4 from Ni and Cheng (2010) shows the relation between jointly connected graphs and its nonzero eigenvalues, and plays a key role in the stability analysis. However, for an adaptive design of uncertain systems, both consensus stability and parameter convergence are considered in this paper. For networks with jointly connected topologies, the parameter convergence analysis is more challenging.
Remark 12 From the proof of Theorem 9, we can see that the consensus result $\lim _{t \rightarrow \infty}\left|x_{i}(t)-x_{0}(t)\right|=0$, $i=1,2, \cdots, N$, is derived without using the PE condition. Therefore, consensus of multi-agent systems will be still reached in networks with jointly connected topologies in the absence of the PE condition. In this case, we certainly would not expect the parameter convergence. In fact, when consensus is reached, $\dot{\bar{\Theta}}$ will be equal to zero and $\bar{\Theta}$ some constant vector. Therefore, no conclusion can be drawn about the behavior of the estimation error $\bar{\Theta}$ in the absence of the PE condition, except that it converges to a constant vector.

\section{Simulations}

In this section, we give two examples to validate our theoretical results. In both examples, we consider a multiagent system consisting of five agents and a leader. The leader agent's unknown velocity dynamic is parameterized as

$$
v_{0}(t)=[\sin (t), \cos (t)] \theta_{0} .
$$

We pick $\theta_{0}=\left[\frac{\sqrt{3}}{2}, \frac{\sqrt{2}}{2}\right]^{T}$. The followers' unknown nonlinear dynamics are parameterized as

$$
f_{i}\left(x_{i}, t\right)=\left[x_{i} \sin (t), x_{i} \cos (t)\right] \theta_{i}, i=1,2, \cdots, 5 .
$$

We select $\theta_{i}=\left[\frac{1}{2}, \frac{1}{2}\right]^{T}, i=1,2, \cdots, 5$, as the true parameters to be estimated. In both examples, all components of the initial state of system are chosen in interval $[-5,5]$ randomly.

Example 13 In this example, a finite automation with set of states $\left\{\bar{G}_{1}, \bar{G}_{2}, \bar{G}_{3}, \bar{G}_{4}\right\}$ is shown in Figure 1 , which represents the discrete states of a network with switching topologies. It starts at the discrete state $\bar{G}_{1}$ and switches every three simulation time steps to the next state according to the state machine in Fig. 1. The proposed adaptive consensus scheme (13) and (15) is realized in this situation and the simulation results are shown in Figures 2 and 3. Figures 2 and 3 show that consensus is reached, parameter convergence is guaranteed under control law (13) and parameter adaptive law (15), respectively.

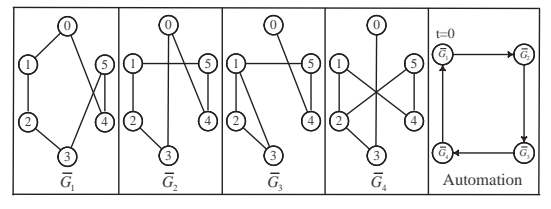

Fig. 1. Switching Graphs

Example 14 In this example, we consider the case of networks with jointly connected topologies. We suppose that the possible interconnected graphs are $\left\{\bar{G}_{1}, \bar{G}_{2}, \bar{G}_{3}, \bar{G}_{4}, \bar{G}_{5}, \bar{G}_{6}\right\}$ which are shown in Figure 4 , and switched every three simulation time steps to the next 


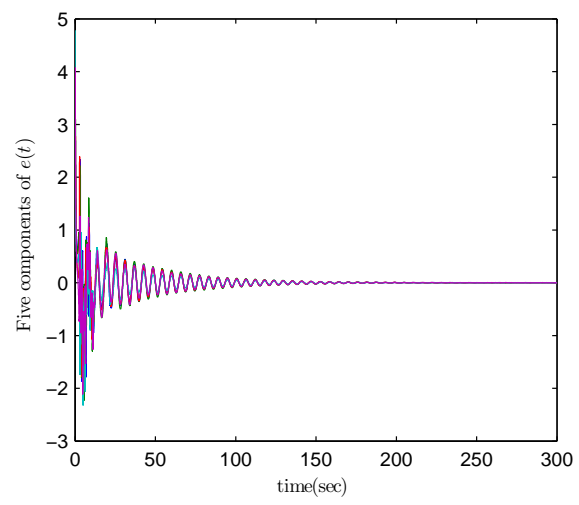

Fig. 2. Consensus is reached applying (13) and (15) in networks with switching topologies.
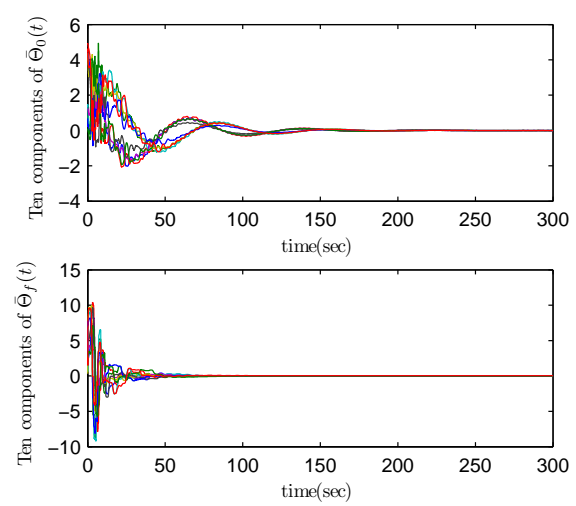

Fig. 3. Parameter convergence is guaranteed applying (13) and (15) in networks with switching topologies.

graph as $\bar{G}_{1} \rightarrow \bar{G}_{2} \rightarrow \bar{G}_{3} \rightarrow \bar{G}_{4} \rightarrow \bar{G}_{5} \rightarrow \bar{G}_{6} \rightarrow \bar{G}_{1} \ldots$. Note that both $\bar{G}_{1} \cup \bar{G}_{2} \cup \bar{G}_{3}$ and $\bar{G}_{4} \cup \bar{G}_{5} \cup \bar{G}_{6}$ are connected, therefore both $\left\{\bar{G}_{1}, \bar{G}_{2}, \bar{G}_{3}\right\}$ and $\left\{\bar{G}_{4}, \bar{G}_{5}, \bar{G}_{6}\right\}$ are jointly connected through some time intervals. Control law (13) and parameter adaptive law (15) are then realized in networks with jointly connected topologies. Simulation results are shown in Figures 5 and 6. Figure 5 and Figure 6 show that consensus is reached and parameter convergence is guaranteed in jointly connected networks under (13) and (15), respectively.

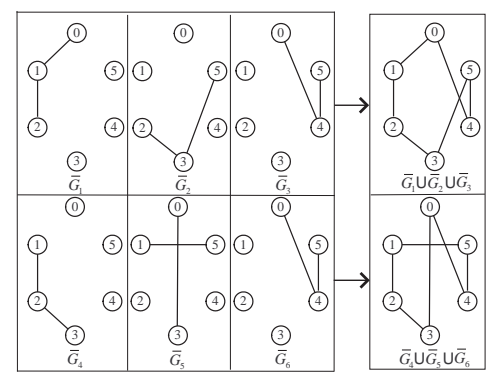

Fig. 4. Jointly connected graphs

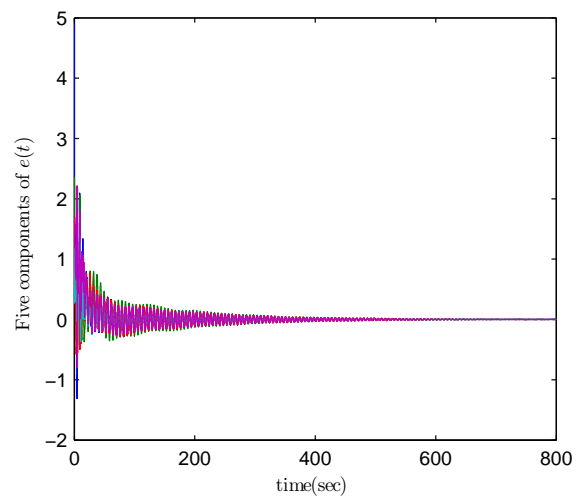

Fig. 5. Consensus is reached applying (13) and (15) in networks with jointly connected topologies.
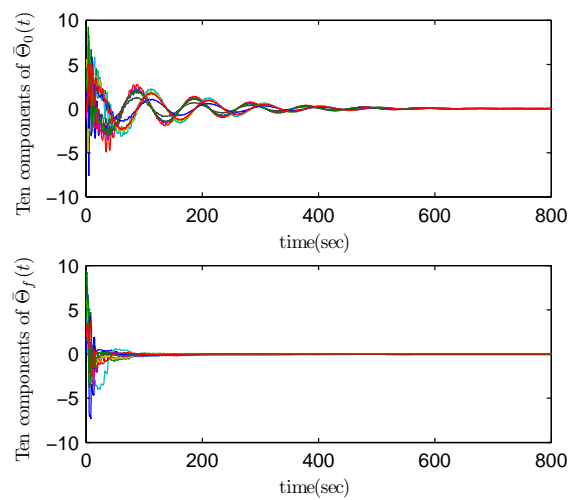

Fig. 6. Parameter convergence is guaranteed applying (13) and (15) in networks with jointly connected topologies.

\section{Conclusions}

In this paper we considered the consensus problem of multi-agents with unknown nonlinear dynamics following a leader also with unknown velocity dynamics. By adaptive control design, consensus with parameter convergence is ensured. Graph theory was used to describe the interconnection topologies. Lyapunov theory and Barbalat's Lemma were employed for stability analysis. The joint connectedness of graphs is a key condition to ensure consensus achievement and the PE condition ensures parameter convergence. Simulations showed the validity of our results.

\section{References}

H. Bai, M. Arcak, and J. T. Wen. Adaptive design for reference velocity recovery in motion coordination. Systems \& Control Letters, 57:602-610, 2008.

H. Bai, M. Arcak, and J. T. Wen. Adaptive motion coordination: Using relative velocity feedback to track a reference velocity. Automatica, 45:1020-1025, 2009.

A. Das and F. L. Lewis. Distributed adaptive control 
for synchronization of unknown nonlinear networked systems. Automatica, 46:2014-2021, 2010.

J. A. Fax and R. M. Murray. Information flow and cooperative control of vehicle formations. IEEE Transactions on Automatic Control, 49(9):1465-1476, September 2004.

C. D. Godsil and G. Royle. Algebraic Graph Theory. Springer, New York, 2001.

Y. Hatano and M. Mesbahi. Agreement over random networks. IEEE Transactions on Automatic Control, 50(11):1867-1872, November 2005.

Y. Hong, J. Hu, and L. Gao. Tracking control for multiagent consensus with an active leader and variable topology. Automatica, 42:1177-1182, 2006.

Z.-G. Hou, L. Cheng, and M. Tan. Decentralized robust adaptive control for the multiagent system consensus problem using neural networks. IEEE Transactions on Systems, Man, and Cybernetics -Part B: Cybernetics, 39(3):636-647, June 2009.

A. Jadbabaie, J. Lin, and A. S. Morse. Coordination of groups of mobile autonomous agents using nearest neighbor rules. IEEE Transactions on Automatic Control, 48(6):988-1001, June 2003.

A. Kashyap, T. Basar, and R. Srikant. Quantized consensus. Automatica, 43:1192-1203, 2007.

S. Khoo, L. Xie, and Z. Man. Robust finite-time consensus tracking algorithm for multirobot systems. IEEE/ASME Transactions on Mechatronics, 14(2): 219-228, April 2009.

Z. Lin, M. Broucke, and B. Francis. Local control strategies for groups of mobile autonomous agents. IEEE Transactions on Automatic Control, 49(4):622-629, April 2004.

R. Marino and P. Tomei. Nonlinear Control Design - Geometric, Adaptive and Robust. Prentice Hall Europe, 1995.

L. Moreau. Stability of multiagent systems with timedependent communication links. IEEE Transactions on Automatic Control, 50(2):169-182, February 2005.

W. Ni and D. Cheng. Leader-following consensus of multi-agent systems under fixed and switching topologies. Systems \& Control Letters, 59:209-217, 2010.

R. Olfati-Saber and R. M. Murray. Consensus problem$\mathrm{s}$ in networks of agents with switching topology and time-delays. IEEE Transactions on Automatic Control, 49(9):1520-1533, September 2004.

W. Ren. Multi-vehicle consensus with a time-varying reference state. Systems \& Control Letters, 56:474483, 2007.

W. Ren and R. W. Beard. Consensus seeking in multiagent systems under dynamically changing interaction topologies. IEEE Transactions on Automatic Control, 50(5):655-661, May 2005.

W. Ren, K. L. Moore, and Y. Chen. High-order and model reference consensus algorithms in cooperative control of multivehicle systems. J. Dyn. Sys., Meas., Control, 129(5):678-688, September 2009.

S. Sastry and M. Bodson. Adaptive Control: Stability, Convergence, and Robustness. Prentice-Hall, New
Jersey, 1989.

A. V. Savkin. Coordinated collective motion of groups of autonomous mobile robots: Analysis of vicsek's model. IEEE Transactions on Automatic Control, 49(6):981983, June 2004.

Y. G. Sun and L. Wang. Consensus of multi-agent systems in directed networks with nonuniform timevarying delays. IEEE Transactions on Automatic Control, 54(7):1607-1613, July 2009.

A. Tahbaz-Salehi and A. Jadbabaie. A necessary and sufficient condition for consensus over random networks. IEEE Transactions on Automatic Control, 53 (3):791-795, April 2008.

W. Yu, G. Chen, and J. Lü. On pinning synchronization of complex dynamical networks. Automatica, 45:429435, 2009a.

W. Yu, J. Lü, G. Chen, Z. Duan, and Q. Zhou. Estimating uncertain delayed genetic regulatory network$\mathrm{s}$ : An adaptive filtering approach. IEEE transactions on automatic control, 54(4):892-897, April 2009b.

W. Zhu and D. Cheng. Leader-following consensus of second-order agents with multiple time-varying delays. Automatica, 46:1994-1999, 2010. 